\title{
Berezinskii-Kosterlitz-Thouless transition in the time-reversal-symmetric Hofstadter-Hubbard model
}

\author{
M. Iskin \\ Department of Physics, Koç University, Rumelifeneri Yolu, 34450 Sarlyer, Istanbul, Turkey
}

(Received 24 October 2017; published 16 January 2018)

\begin{abstract}
Assuming that two-component Fermi gases with opposite artificial magnetic fields on a square optical lattice are well described by the so-called time-reversal-symmetric Hofstadter-Hubbard model, we explore the thermal superfluid properties along with the critical Berezinskii-Kosterlitz-Thouless (BKT) transition temperature in this model over a wide range of its parameters. In particular, since our self-consistent BCS-BKT approach takes the multiband butterfly spectrum explicitly into account, it unveils how dramatically the interband contribution to the phase stiffness dominates the intraband one with an increasing interaction strength for any given magnetic flux.
\end{abstract}

DOI: 10.1103/PhysRevA.97.013618

\section{INTRODUCTION}

The phase stiffness, also known as the helicity modulus, measures the response of a system in an ordered phase to a twist of the order parameter [1] and it is directly linked to the superfluid (SF) density of the superconducting systems [2,3]. In its most familiar form, the conventional expression for the elements of the phase stiffness tensor can be written as [4]

$$
\begin{aligned}
D_{\mu \nu}^{\text {one band }}= & \frac{1}{\mathcal{V}_{d}} \sum_{\mathbf{k}}\left\{\frac{\partial^{2} \xi_{\mathbf{k}}}{\partial k_{\mu} \partial k_{\nu}}\left[1-\frac{\xi_{\mathbf{k}}}{E_{\mathbf{k}}} \tanh \left(\frac{E_{\mathbf{k}}}{2 k_{B} T}\right)\right]\right. \\
& \left.-\frac{1}{2 k_{B} T}\left(\frac{\partial \xi_{\mathbf{k}}}{\partial k_{\mu}} \frac{\partial \xi_{\mathbf{k}}}{\partial k_{\nu}}\right) \operatorname{sech}^{2}\left(\frac{E_{\mathbf{k}}}{2 k_{B} T}\right)\right\},
\end{aligned}
$$

where $\mathcal{V}_{d}$ is the volume element and $\mathbf{k}$ is the wave vector in $d$ spatial dimensions, $k_{v}$ with $v \equiv\{x, y, \ldots\}$ is the projection of $\mathbf{k}, \xi_{\mathbf{k}}=\varepsilon_{\mathbf{k}}-\mu$ is the single-particle dispersion relation shifted by the chemical potential, $E_{\mathbf{k}}=\sqrt{\xi_{\mathbf{k}}^{2}+\left|\Delta_{\mathbf{k}}\right|^{2}}$ is the quasiparticle dispersion relation with the order parameter $\Delta_{\mathbf{k}}$, $k_{B}$ is the Boltzmann constant, and $T$ is the temperature. Here $\varepsilon_{\mathbf{k}}$ is assumed to be quite general and not limited with the usual quadratic dependence on $k_{v}$. In particular, this tensor plays a special role in two dimensions for which it appears explicitly in the universal Berezinskii-Kosterlitz-Thouless (BKT) relation determining the critical SF transition temperature $T_{\mathrm{BKT}}$ [2-4]. This is a topological phase transition characterized by the binding (unbinding) of two vortices with opposite circulations, i.e., the so-called vortex-antivortex pairs, below (above) $T_{\mathrm{BKT}}$ with algebraically (exponentially) decaying spatial correlations [5-7]. For instance, one of the immediate manifestations of Eq. (1) is that it rules out the possibility of superfluidity in systems with a nearly flat $\mathbf{k}$-space dispersion, i.e., the SF density and current are identically zero since the particles are strictly immobile in a flatband with $\xi_{\mathbf{k}} \approx \xi_{0}$ for all $\mathbf{k}$.

Motivated by the experimental advances with cold Fermi gases, the calculation of $D_{\mu \nu}$ has recently been extended to a class of multiband Hamiltonians that are characterized by a single mean-field order parameter $\Delta$ with a uniform spread in real space and that exhibit time-reversal $\mathcal{T}$ symmetry [8-11]. It has been found that, in addition to the intraband contribution to
$D_{\mu \nu}$ that has exactly the same form as the one given in Eq. (1) for each single-particle band, the interband contribution may also be necessary for a proper description of the multiband systems. For instance, in marked contrast with the single flatband systems, it turns out that superfluidity may prevail in a flatband in the presence of other bands as a result of the interband tunnelings [8,9]. See also the related discussion of two-band superconductivity in graphene for a resolution of the superconductivity without supercurrent controversy in the vicinity of its Dirac points $[12,13]$.

In view of the recent realization of the Hofstadter-Hubbard model with $\mathcal{T}$ symmetry [14,15] and the forthcoming experiments, here we study $T_{\mathrm{BKT}}$ in this model and address the interplay between the intraband and interband contributions to the phase stiffness in the presence of a multiband butterfly spectrum. Despite our naive expectations, we find that the maximum $T_{\mathrm{BKT}} \approx 0.253 t / k_{B}$ is attained for the no-flux limit at $\mu=$ 0 when the interaction strength is around $U \sim 3.75 t$. Here $t$ is the hopping strength. In addition, one of the highlights of this paper is that increasing the interaction strength always shifts the relative importance of the intraband and interband contributions in overwhelming favor of the latter and that the proper description of the Cooper molecules requires an indiscriminate account of both contributions in the strong-coupling limit.

The remainder of this paper is organized as follows. After a short overview of the Hofstadter model with $\mathcal{T}$ symmetry in Sec. II A, we introduce the self-consistent BCS-BKT formalism in Sec. IIB, with the multiband generalization of the phase stiffness detailed in Sec. II C. We discuss the analytically tractable strong coupling or molecular limit in Sec. IID and then our numerical results are presented in Sec. III. We end the paper with a brief summary of our conclusions in Sec. IV.

\section{THEORETICAL FRAMEWORK}

Assuming that the tight-binding approximation is a viable description of the kinematics of a two-component Fermi gas on an optical lattice, we start with the single-particle Hamiltonian $H_{0}=-\sum_{i j} c_{i}^{\dagger} t_{i j} c_{j}$, where $c_{i}^{\dagger}\left(c_{i}\right)$ creates (annihilates) a spinless fermion at site $i$ so that $t_{i j}=t_{j i}^{*}$ is the element 
of the hopping matrix from site $j$ to $i$. This model also offers a convenient way to incorporate the effects of additional gauge fields, e.g., an external magnetic field $\mathbf{B}(\mathbf{r})=\nabla \times \mathbf{A}(\mathbf{r})$ may be taken into account via the minimal coupling, i.e., $t_{i j} \rightarrow t_{i j} e^{i 2 \pi \phi_{i j}}$, leading to an additional phase factor $\phi_{i j}=$ $\left(1 / \phi_{0}\right) \int_{\mathbf{r}_{j}}^{\mathbf{r}_{i}} \mathbf{A}(\mathbf{r}) \cdot d \mathbf{r}$ in the hopping matrix. Here $\phi_{0}$ is the flux quantum and $\mathbf{A}(\mathbf{r})$ is the magnetic vector potential. In a broader context, $\mathbf{A}(\mathbf{r})$ could be any gauge field, including the artificial ones created in atomic systems.

In this paper we are interested in a square lattice lying in the entire $(x, y)$ plane, which is under the influence of a spatially uniform magnetic field $B(\mathbf{r})=B$ pointing along the perpendicular $z$ axis. Such a setting can be represented by $\mathbf{A}(\mathbf{r})=(0, B x, 0)$ in the Landau gauge without losing generality. Thus, for a given flux quantum per unit cell $\alpha=B a^{2} / \phi_{0}$, with $a$ the lattice spacing, the particle gains an Aharonov-Bohm phase $e^{i 2 \pi \alpha}$ after traversing a loop around the unit cell. Next we consider the original Hofstadter model [16] and allow the particle to hop back and forth between only the nearestneighbor sites, which by itself gives rise to one of the most fascinating single-particle energy spectra in nature.

\section{A. Hofstadter butterfly}

When the flux $\alpha=p / q$ corresponds precisely to the ratio of two relatively prime numbers $p$ and $q$, one can simplify the single-particle problem considerably by switching to the reciprocal (k) space representation and making use of the new translational symmetry [16]. That is, since the $B$ field enlarges the unit cell by a factor of $q$ in the $x$ direction, the first magnetic Brillouin zone (MBZ) is reduced to $-\pi / q a \leqslant k_{x}<\pi / q a$ and $-\pi / a \leqslant k_{y}<\pi / a$. This not only splits the tight-binding $s$ band of the flux-free system, i.e., $\varepsilon_{\mathbf{k}}=-2 t \cos \left(k_{x} a\right)-$ $2 t \cos \left(k_{y} a\right)$, into $q$ subbands for a given $\alpha=p / q$, but the competition between the magnetic length scale, i.e., the cyclotron radius, and the periodicity of the lattice potential also produces a very complicated energy $\varepsilon$ versus $p / q$ landscape with an underlying fractal pattern. As the overall landscape bears a resemblance to the shape of a butterfly, the spectrum is usually referred to as the Hofstadter butterfly in the literature [16].

For a given $\alpha=p / q$, the multiband spectrum can be obtained by solving the Schrödinger equation $\mathbb{H}_{0 \mathbf{k}}|n \mathbf{k}\rangle=$ $\varepsilon_{n \mathbf{k}}|n \mathbf{k}\rangle$ in $\mathbf{k}$ space, where $n=0, \ldots, q-1$ labels the subbands starting from the lowest-energy branch. This leads to $\sum_{j=0}^{q-1} H_{0 \mathbf{k}}^{i j} g_{n \mathbf{k}}^{j}=\varepsilon_{n \mathbf{k}} g_{n \mathbf{k}}^{i}$, where $g_{n \mathbf{k}}^{i}$ is the $i=0,1, \ldots, q-1$ th component of the $n=0,1, \ldots, q-1$ th eigenvector of the single-particle problem with energy $\varepsilon_{n \mathbf{k}}$. Here the Fourierexpansion coefficient $c_{i \mathbf{k}}$ of the site operator can be written in terms of the band operators $d_{n \mathbf{k}}$ as $c_{i \mathbf{k}}=\sum_{n=0}^{q-1} g_{n \mathbf{k}}^{i} d_{n \mathbf{k}}$. Thus, for a given $\mathbf{k}$ in the MBZ, the spectrum is determined by solving the equation

$$
\left(\begin{array}{cccccc}
B_{\mathbf{k}}^{0} & C_{\mathbf{k}} & 0 & \ldots & 0 & C_{\mathbf{k}}^{*} \\
C_{\mathbf{k}}^{*} & B_{\mathbf{k}}^{1} & C_{\mathbf{k}} & 0 & \ldots & 0 \\
0 & \ddots & \ddots & \ddots & 0 & \vdots \\
\vdots & 0 & C_{\mathbf{k}}^{*} & B_{\mathbf{k}}^{j} & C_{\mathbf{k}} & 0 \\
0 & \ddots & 0 & \ddots & \ddots & \ddots \\
C_{\mathbf{k}} & 0 & \cdots & 0 & C_{\mathbf{k}}^{*} & B_{\mathbf{k}}^{q-1}
\end{array}\right)\left(\begin{array}{c}
g_{n \mathbf{k}}^{0} \\
\vdots \\
g_{n \mathbf{k}}^{j-1} \\
g_{n \mathbf{k}}^{j} \\
g_{n \mathbf{k}}^{j+1} \\
\vdots \\
g_{n \mathbf{k}}^{q-1}
\end{array}\right)=0,
$$

where $\quad B_{\mathbf{k}}^{j}=-2 t \cos \left(k_{y} a+2 \pi \alpha j\right)-\varepsilon_{n \mathbf{k}} \quad$ with $\quad j=$ $0,1, \ldots, q-1$ and $C_{\mathbf{k}}=-t e^{i k_{x} a}$. We note that while the orthonormalization condition $\langle n \mathbf{k} \mid m \mathbf{k}\rangle=\delta_{n m} \quad$ leads to $\quad \sum_{j=0}^{q-1} g_{n \mathbf{k}}^{j *} g_{m \mathbf{k}}^{j}=\delta_{n m}$, the completeness relation $\sum_{n=0}^{q-1}|n \mathbf{k}\rangle\langle n \mathbf{k}|=\mathbb{I}$ leads to $\sum_{n=0}^{q-1} g_{n \mathbf{k}}^{i *} g_{n \mathbf{k}}^{j}=\delta_{i j}$ for any given $\mathbf{k}$ state, both of which are used throughout the paper in simplifying the self-consistency equations.

The butterfly spectrum exhibits a number of symmetries. First of all, it preserves the inversion symmetry in $\mathbf{k}$ space, i.e., $\varepsilon_{n \mathbf{k}}=\varepsilon_{n,-\mathbf{k}}$, as a direct manifestation of the gauge invariance in a uniform flux. In addition, not only is it symmetric around $\varepsilon=0$ for a given flux, i.e., $\varepsilon_{n \mathbf{k}}(\alpha)=-\varepsilon_{q-1-n,-\mathbf{k}}(\alpha)$, due to the particle-hole symmetry of $H_{0}$ on a bipartite lattice, but it is also mirror symmetric around $\alpha=1 / 2$ for a given $|n \mathbf{k}\rangle$ state, i.e., $\varepsilon_{n \mathbf{k}}(\alpha)=\varepsilon_{n \mathbf{k}}(1-\alpha)$ for $0 \leqslant \alpha \leqslant 1$. The latter relation suggests that the flux-free $\alpha=0$ system is exactly equivalent to the $\alpha=1$ case and hence $\alpha=1 / 2$ corresponds to the maximally attainable flux [16]. Furthermore, when $q$ is an even denominator, combination of the inversion and particle-hole symmetries implies the condition $\varepsilon_{q / 2-1, \mathbf{k}}=-\varepsilon_{q / 2, \mathbf{k}}$, from which we infer that the centrally symmetric bands $n=q / 2-1$ and $n=q / 2$ have degenerate $\mathbf{k}$ states with $\varepsilon=0$. It turns out that these central bands contain $q$ Dirac cones with $q$ zero-energy touchings in the first MBZ and therefore are not separated by a bulk energy gap. For example, setting $p / q=1 / 2$, we obtain $\varepsilon_{n \mathbf{k}}=(-1)^{n+1} 2 t \sqrt{\cos ^{2}\left(k_{x} a\right)+\cos ^{2}\left(k_{y} a\right)}$ for the $n=0$ and $n=1$ bands, where $g_{n \mathbf{k}}^{0} / g_{n \mathbf{k}}^{1}=-\cos \left(k_{y} a\right) /\left[\cos \left(k_{y} a\right)+\right.$ $\left.(-1)^{n+1} \sqrt{\cos ^{2}\left(k_{x} a\right)+\cos ^{2}\left(k_{y} a\right)}\right]$ together with $\left|g_{n \mathbf{k}}^{0}\right|^{2}+$ $\left|g_{n \mathbf{k}}^{1}\right|^{2}=1$. The locations of the Dirac points are $k_{x}=-\pi / 2 a$ and $k_{y}= \pm \pi / 2 a$. Since the $\alpha=1 / 2$ case is a nontrivial yet an analytically tractable one, we often use it as one of the ultimate benchmarks for the accuracy of our numerical calculations.

Our primary interest in this paper is the superfluidity of a spin-1/2 Fermi gas on a square lattice that is experiencing an equal but opposite magnetic field for its spin components [17-19], i.e., $\alpha_{\uparrow}=-\alpha_{\downarrow}=\alpha$. This restores the $\mathcal{T}$ symmetry in the system in such a way that the solutions of the Hofstadter model for a $\downarrow$ particle can be written in terms of the $\uparrow$ ones (given above) as follows: $\varepsilon_{\downarrow n \mathbf{k}}=\varepsilon_{\uparrow n,-\mathbf{k}}$ and $g_{\downarrow n \mathbf{k}}^{j}=g_{\uparrow n,-\mathbf{k}}^{j^{*}}$. More importantly, the self-consistent mean-field theory of such a time-reversal-symmetric Hofstadter-Hubbard model [18,19] turns out to be dramatically simpler to implement than that of the usual Hofstadter-Hubbard model [20,21], as we discuss next.

\section{B. Self-consistent BCS-BKT theory}

Having an equal number of $\uparrow$ and $\downarrow$ particles that are interacting with on-site and attractive interactions in mind, we have recently shown that the mean-field Hamiltonian can be simply written as $[18,19]$

$$
H=\sum_{\sigma n \mathbf{k}} \xi_{n \mathbf{k}} d_{\sigma n \mathbf{k}}^{\dagger} d_{\sigma n \mathbf{k}}-\Delta \sum_{n \mathbf{k}}\left(d_{\uparrow n \mathbf{k}}^{\dagger} d_{\downarrow n,-\mathbf{k}}^{\dagger}+\text { H.c. }\right)
$$

in $\mathbf{k}$ space. Here $H$ is given up to a constant $M \Delta^{2} / U$ term, where $M=\mathcal{A} / a^{2}$ is the number of lattice sites with $\mathcal{A}$ the area of the system, $\xi_{n \mathbf{k}}=\varepsilon_{n \mathbf{k}}-\mu$ is the butterfly spectrum $\varepsilon_{n \mathbf{k}}$ shifted by the chemical potential $\mu$, and $\Delta=$ 
$(U / M) \sum_{n \mathbf{k}}\left\langle d_{\downarrow n,-\mathbf{k}} d_{\uparrow n \mathbf{k}}\right\rangle$ is the order parameter characterizing a spatially uniform SF phase. In addition, $U \geqslant 0$ is the strength of the interparticle interactions, $\langle\cdots\rangle$ denotes the thermal average, H.c. is the Hermitian conjugate, and $\Delta$ is assumed to be real without losing generality. In contrast to the usual Hofstadter-Hubbard model where the competing vortex-latticelike SF phases involve both intra- and interband Cooper pairings with nontrivial sets of finite center-of-mass momenta $\mathbf{K}$ and hence require $q \times q$ order parameters [20,21], here the energetically more favorable mean-field solution is in essence a matter of the superfluidity of intraband Cooper pairs with $\mathbf{K}=\mathbf{0}$ only $[18,19]$. This is simply because, as the $\mathcal{T}$ symmetry guarantees the existence of a $\downarrow$ partner in state $|n,-\mathbf{k}\rangle$ for every $\uparrow$ fermion in state $|n \mathbf{k}\rangle$, the spatially uniform SF solution allows all particles to take advantage of the attractive potential by making $\uparrow \downarrow$ Cooper pairs with $\mathbf{K}=\mathbf{0}$. We also emphasize that the disappearance of all of the interband pairing terms from the mean-field Hamiltonian is a direct consequence of the uniform SF phase with $\mathcal{T}$ symmetry.

Given the quadratic Hamiltonian, minimization of the corresponding thermodynamic potential with respect to $\Delta$, together with the number equation $N=\sum_{\sigma n \mathbf{k}}\left\langle d_{\sigma n \mathbf{k}}^{\dagger} d_{\sigma n \mathbf{k}}\right\rangle$ that is controlled by $\mu$, leads to a closed set of self-consistency equations that are analytically tractable. For instance, a compact way to express these mean-field equations is ${ }^{1}[18,19]$

$$
\begin{gathered}
1=\frac{U}{2 M} \sum_{n \mathbf{k}} \frac{\mathcal{X}_{n \mathbf{k}}}{E_{n \mathbf{k}}}, \\
F=1-\frac{1}{M} \sum_{n \mathbf{k}} \frac{\mathcal{X}_{n \mathbf{k}}}{E_{n \mathbf{k}}} \xi_{n \mathbf{k}},
\end{gathered}
$$

where $\mathcal{X}_{n \mathbf{k}}=\tanh \left(E_{n \mathbf{k}} / 2 k_{B} T\right)$ is a thermal factor with $k_{B}$ the Boltzmann constant and $T$ the temperature, $E_{n \mathbf{k}}=\sqrt{\xi_{n \mathbf{k}}^{2}+\Delta^{2}}$ is the energy spectrum of the quasiparticles arising from band $n$, and the particle filling $0 \leqslant F=N / M \leqslant 2$ corresponds to the total number of particles per site. Thus, we use Eqs. (4) and (5) to determine $\Delta$ and $\mu$ for any given set of $U, F, T$, and $\alpha$ parameters.

Since neither the amplitude nor the phase fluctuations of the SF order parameter are included in the mean-field theory, while the self-consistent solutions of Eqs. (4) and (5) are a reliable description of the ground state at $T=0$ for all $U$ values, the theory works reasonably well at finite temperatures $T \lesssim T_{\mathrm{BCS}} \ll t / k_{B}$ as long as $U \lesssim t$ is weak. ${ }^{2}$ Here $T_{\mathrm{BCS}}$ is the critical BCS transition temperature that is determined by setting $\Delta \rightarrow 0$. However, as the role played by the temporal phase fluctuations increases dramatically with stronger $U \gtrsim t$ values, the mean-field theory becomes gradually insufficient, failing eventually at capturing the finite-temperature correlations of the SF phase in the strong-coupling or molecular limit

\footnotetext{
${ }^{1}$ The extra $q$ factor appearing in the denominator of Eq. (3) is a typographical error in Ref. [18].

${ }^{2}$ Even though the mean-field BCS theory breaks down when $T>$ $T_{\mathrm{BKT}}$, we find that $\Delta=\sqrt{3} \Delta_{0} \sqrt{1-T / T_{\mathrm{BCS}}}$ works extremely well for $T \approx T_{\mathrm{BCS}}$. Here $\Delta_{0}$ is the $T=0$ order parameter and the prefactor $\sqrt{3}$ is quite robust in the entire parameter range. See also [19] for a similar observation.
}

even though $T \ll T_{\mathrm{BCS}}[22,23]$. In the $U \gg t$ limit, we note that the mean-field $T_{\mathrm{BCS}}$ is directly proportional to the binding energy $U$ of the two-body bound state in vacuum and therefore it characterizes the pair formation temperature of the Cooper molecules. Thus, away from the weak-coupling limit, $T_{\mathrm{BCS}}$ has obviously nothing to do with the critical SF transition temperature of the system, for which the phase coherence is known to be established at a much lower temperature.

Taking only the phase fluctuations into account in our two-dimensional model characterized by a single SF order parameter, the critical SF transition temperature is determined by the universal BKT relation ${ }^{3}$ [4-7]

$$
k_{B} T_{\mathrm{BKT}}=\frac{\pi}{8} D_{0}\left(T_{\mathrm{BKT}}\right),
$$

where $D_{0}$ is the isotropic measure of the $2 \times 2$ phase stiffness tensor, i.e., $D_{\mu \nu}=D_{0} \delta_{\mu \nu}$ with $(\mu, v) \equiv\{x, y\}$. Similar to the usual Hubbard model with a single SF order parameter [4] and due to the time-reversal symmetry of the current model, the elements $D_{\mu \nu}$ are identified by making an analogy with the effective phase-only $X Y$ Hamiltonian $[8,10]$, where $H_{X Y}=$ $(1 / 8) \int d x \int d y \sum_{\mu \nu} \partial_{\mu} \theta_{\mathbf{r}} D_{\mu \nu} \partial_{\nu} \theta_{\mathbf{r}}$ under the assumption that $\Delta_{\mathbf{r}}=\Delta e^{i \theta_{\mathbf{r}}}$. Setting $\theta_{\mathbf{r}}=\mathbf{K} \cdot \mathbf{r}$ for a spatially uniform condensate density with $\hbar \mathbf{K}$ the pair momentum, we note that $H_{X Y}=D_{0} \mathcal{A} K^{2} / 4=m_{0} \mathcal{A} \rho_{s} v^{2} / 2$, where $\mathbf{v}=\hbar \mathbf{K} / 2 m_{0}$ is the velocity of the SF pairs with $m_{0}$ the mass of the particles and $\rho_{s}=m_{0} D_{0} / \hbar^{2}$ is the density of the SF particles. Thus, the phase stiffness of a SF is essentially equivalent to its SF density. Here the factor $m_{0} \rho_{s}$ is often called the SF mass density of the system.

We note that since $T_{\mathrm{BCS}}$ is determined by the BCS condition $\Delta \rightarrow 0$ and a finite $T_{\mathrm{BKT}}$ requires a finite $\Delta$ by definition, Eq. (6) already puts $T_{\mathrm{BCS}}$ as the upper bound on $T_{\mathrm{BKT}}$ for any $U \neq 0$. It turns out that while $T_{\mathrm{BKT}} \sim t^{2} / k_{B} U \ll T_{\mathrm{BCS}}$ in the $U / t \gg 1$ limit [22,23], $T_{\mathrm{BKT}} \rightarrow T_{\mathrm{BCS}}$ in the opposite $U / t \lesssim 1$ limit where the rate $D_{0} / t \rightarrow 0$ is the same as $\Delta / t \rightarrow 0$ only when $U / t \rightarrow 0$. In fact, we find in Sec. III that the maximum $T_{\mathrm{BKT}} \approx 0.253 t / k_{B}$ is attained for $q=1$ at $\mu=0$ when $U \sim$ $3.75 t$.

Next we justify that the self-consistent solutions of Eqs. (4)(6) for $\Delta, \mu$, and $T_{\mathrm{BKT}}$, along with the proper multiband generalization [10] of $D_{\mu \nu}$ given below, are a reliable description of the SF transition temperature $T_{\mathrm{BKT}}$ for any given set of $U, F$, and $\alpha$ parameters. We note in passing that the self-consistent BCS-BKT approach amounts to be the simultaneous solutions of BCS mean-field equations and the universal BKT relation, i.e., the phase fluctuations are only taken into account by the latter via the analogy with the underlying $X Y$ model. While this simple description is known to be quite accurate for the weak-coupling BCS and strong-coupling molecular limits, it provides a qualitative but reliable picture of the crossover regime.

\section{Phase stiffness}

As an alternative to the expression given in Ref. [10], a compact way to write the elements of the phase stiffness

\footnotetext{
${ }^{3}$ If $D_{\mu \nu}$ is not isotropic then the universal relation becomes $k_{B} T_{\mathrm{BKT}}=(\pi / 8) \operatorname{det} \mathbb{D}$.
} 
tensor is

$$
\begin{aligned}
D_{\mu \nu}= & \frac{\Delta^{2}}{\mathcal{A}} \sum_{n m \mathbf{k}}\left(\frac{\mathcal{X}_{n \mathbf{k}}}{E_{n \mathbf{k}}}-\frac{\mathcal{X}_{n \mathbf{k}}-\mathcal{X}_{m \mathbf{k}}}{E_{n \mathbf{k}}-E_{m \mathbf{k}}}\right) \\
& \times \frac{2 Q_{\mu \nu}^{n m \mathbf{k}}}{E_{m \mathbf{k}}\left(E_{n \mathbf{k}}+E_{m \mathbf{k}}\right)},
\end{aligned}
$$

where the independent $n$ and $m$ summations run over all bands, $\mathcal{X}_{n \mathbf{k}}=\tanh \left(E_{n \mathbf{k}} / 2 k_{B} T\right)$ is the thermal factor, and the coefficient $Q_{\mu \nu}^{n m \mathbf{k}}=\operatorname{Re}\left[\left\langle n \mathbf{k}\left|\partial \mathbb{H}_{0 \mathbf{k}} / \partial k_{\mu}\right| m \mathbf{k}\right\rangle\left\langle m \mathbf{k}\left|\partial \mathbb{H}_{0 \mathbf{k}} / \partial k_{\nu}\right| n \mathbf{k}\right\rangle\right]$ is directly related to the details of the band geometry of the single-particle problem. ${ }^{4}$ For instance, since $D_{\mu \nu}$ is isotropic in space for spatially uniform SFs, we have $Q_{\mu \nu}^{n m \mathbf{k}}=Q_{0}^{n m \mathbf{k}} \delta_{\mu \nu}$ with the particular coefficient $Q_{0}^{n m \mathbf{k}}=4 t^{2} a^{2} \mid \sum_{j=0}^{q-1} \sin \left(k_{y} a+\right.$ $2 \pi \alpha j)\left.g_{n \mathbf{k}}^{j *} g_{m \mathbf{k}}^{j}\right|^{2}$ obtained for our model Hamiltonian given in Eq. (2). Furthermore, by defining $D_{\mu \nu}=D_{\mu \nu}^{\text {intra }}+D_{\mu \nu}^{\text {inter }}$, we distinguish the intraband contribution of the phase stiffness from the interband contribution, which are based, respectively, on whether $n=m$ or not in Eq. (7). Such an association proves to be illuminating in some of our analysis given below.

First, let us show that the intraband contribution of Eq. (7) corresponds precisely to the conventional expression given in Eq. (1). Setting $n \rightarrow m$ for the intraband contribution, the second term in large parentheses implies a derivative such that $d \tanh (a x) / d x=a \operatorname{sech}^{2}(a x)$ and the coefficient $Q_{\mu \nu}^{n n \mathbf{k}}=\left(\partial \varepsilon_{n \mathbf{k}} / \partial k_{\mu}\right)\left(\partial \varepsilon_{n \mathbf{k}} / \partial k_{\nu}\right)$ depends only on the spectrum. After plugging them into Eq. (7), we rearrange the intraband contribution into two pieces as $D_{\mu \nu}^{\text {intra }}=(1 / \mathcal{A}) \sum_{n \mathbf{k}}\left(\partial \xi_{n \mathbf{k}} /\right.$ $\left.\partial k_{\mu}\right) \partial\left(\xi_{n \mathbf{k}} \mathcal{X}_{n \mathbf{k}} / E_{n \mathbf{k}}\right) / \partial k_{v}-\left(1 / 2 \mathcal{A} k_{B} T\right) \sum_{n \mathbf{k}} \operatorname{sech}^{2}\left(E_{n \mathbf{k}} / 2 k_{B} T\right)$ $\left(\partial \xi_{n \mathbf{k}} / \partial k_{\mu}\right)\left(\partial \xi_{n \mathbf{k}} / \partial k_{v}\right)$. Since the latter piece already appears in the conventional expression, next we recast the first piece into two summations as $(1 / \mathcal{A}) \sum_{n \mathbf{k}} \partial\left[\left(\partial \xi_{n \mathbf{k}} / \partial k_{\mu}\right)\right.$ $\left.\xi_{n \mathbf{k}} \mathcal{X}_{n \mathbf{k}} / E_{n \mathbf{k}}\right] / \partial k_{v}-(1 / \mathcal{A}) \sum_{n \mathbf{k}}\left(\partial^{2} \xi_{n \mathbf{k}} / \partial k_{\mu} \partial k_{v}\right) \xi_{n \mathbf{k}} \mathcal{X}_{n \mathbf{k}} / E_{n \mathbf{k}}$ Note here that while the first summation integrates to zero as the derivatives $\partial \xi_{n \mathbf{k}} / \partial k_{\mu}$ vanish at the MBZ boundaries, the second summation is equivalent to $(1 / \mathcal{A}) \sum_{n \mathbf{k}}(1-$ $\left.\xi_{n \mathbf{k}} \mathcal{X}_{n \mathbf{k}} / E_{n \mathbf{k}}\right)\left(\partial^{2} \xi_{n \mathbf{k}} / \partial k_{\mu} \partial k_{\nu}\right)$, as the additional summation $(1 / \mathcal{A}) \sum_{n \mathbf{k}} \partial^{2} \xi_{n \mathbf{k}} / \partial k_{\mu} \partial k_{v}=0$ integrates to zero for the same reason given just above. Thus, the intraband contribution of Eq. (7) is precisely the conventional expression in disguise.

For a more explicit demonstration, we substitute $\varepsilon_{\mathbf{k}}=$ $\hbar^{2} k^{2} / 2 m_{0}$ for the dispersion relation in Eq. (7) and obtain the SF stiffness of a single-band continuum system at $T=0$ as $D_{\mu \nu}=\left(\hbar^{2} \Delta^{2} / m_{0}^{2} \mathcal{A}\right) \sum_{\mathbf{k}} k_{\mu} k_{\nu} / E_{\mathbf{k}}^{3}$, leading to $D_{0}=(\mu+$ $\left.\sqrt{\mu^{2}+\Delta^{2}}\right) / 2 \pi$ for any $\Delta \neq 0$. After plugging the meanfield solutions $\Delta=\sqrt{2 \varepsilon_{b} \varepsilon_{F}}$ and $\mu=\varepsilon_{F}-\varepsilon_{b} / 2$ into this expression, where $\varepsilon_{F}=\hbar^{2} k_{F}^{2} / 2 m_{0}$ is the Fermi energy and $\varepsilon_{b} \geqslant 0$ is the two-body binding energy in vacuum, i.e., $1=$ $(U / M) \sum_{\mathbf{k}} 1 /\left(2 \varepsilon_{\mathbf{k}}+\varepsilon_{b}\right)$, we obtain $D_{0}=\hbar^{2} \rho_{F} / m_{0}$ for any $\Delta \neq 0$. Here $\rho_{F}=N / \mathcal{A}=k_{F}^{2} / 2 \pi$ is the total density of particles with $k_{F}$ the Fermi wave vector. Alternatively, this result follows immediately from the $T=0$ limits of the conventional expression $D_{0}=\left(\hbar^{2} / m_{0} \mathcal{A}\right) \sum_{\mathbf{k}}\left(1-\xi_{\mathbf{k}} / E_{\mathbf{k}}\right)$, given in Eq. (1), together with the number equation $N=\sum_{\sigma \mathbf{k}}\left(1 / 2-\xi_{\mathbf{k}} / 2 E_{\mathbf{k}}\right)$. Thus, $D_{0}$ suggests that the entire continuum Fermi gas becomes

\footnotetext{
${ }^{4}$ Application of this general expression to the superconducting graphene [10,19] reproduces the results of Ref. [13].
}

a SF for any $\Delta \neq 0$, i.e., $\rho_{s}=\rho_{F}$ at $T=0$ as soon as $U>0$. By making an analogy with this continuum result, we identify the SF density of particles for the lattice model in Sec. IID.

Second, in the case of two-band SFs, e.g., when $q=2$ in our model as discussed in Sec. II A, it can be explicitly shown that [10] the integrand of the interband contribution is linked to the total quantum metric of the bands $\sum_{n= \pm} f_{\mu \nu}^{n \mathbf{k}}$ with $f_{\mu \nu}^{+, \mathbf{k}}=$ $f_{\mu \nu}^{-, \mathbf{k}}$, where $f_{\mu \nu}^{n \mathbf{k}}=\operatorname{Re}\left[\left\langle\partial_{k_{\mu}} n \mathbf{k}|(\mathbb{I}-|n \mathbf{k}\rangle\langle n \mathbf{k}|)| \partial_{k_{v}} n \mathbf{k}\right\rangle\right] \quad$ or equivalently $f_{\mu \nu}^{n \mathbf{k}}=\sum_{m\{\neq n\}} Q_{\mu \nu}^{n m \mathbf{k}} /\left(\varepsilon_{n \mathbf{k}}-\varepsilon_{m \mathbf{k}}\right)^{2}$ is the quantum metric of band $n$ in general. Note that, as the eigenfunctions $g_{n \mathbf{k}}^{j}$ are only determined up to a random phase factor for a given $|n \mathbf{k}\rangle$ in a computer program, their partial derivatives contain indefinite factors, making the former expression unsuitable for numerical computation. This ambiguity is nicely resolved by transforming the derivatives to the Hamiltonian matrix in the latter expression.

Third, the general expression given in Eq. (7) acquires a much simpler form at sufficiently low temperatures when $k_{B} T \ll \Delta$. Assuming this is the case, we set $\mathcal{X}_{n \mathbf{k}} \rightarrow 1$ for every $|n \mathbf{k}\rangle$ state and obtain

$$
D_{\mu \nu}=\frac{\Delta^{2}}{\mathcal{A}} \sum_{n m \mathbf{k}} \frac{2 Q_{\mu \nu}^{n m \mathbf{k}}}{E_{n \mathbf{k}} E_{m \mathbf{k}}\left(E_{n \mathbf{k}}+E_{m \mathbf{k}}\right)} .
$$

To be exact, this expression is precisely the $T=0$ limit of Eq. (7). However, it is also valid for all $T \leqslant T_{\mathrm{BKT}}$ in the molecular limit, since $k_{B} T_{\mathrm{BKT}} \sim t^{2} / U$ when $U / t \gg 1$ and hence $k_{B} T_{\mathrm{BKT}} \ll t \ll \Delta$ is well founded.

\section{Molecular limit}

In the $\Delta \gg t$ or equivalently $U \gg t$ limit of tightly bound Cooper molecules [22,23], Eqs. (4) and (5) give $\Delta=$ $(U / 2) \sqrt{F(2-F)}$ and $\mu=-(U / 2)(1-F)$, so $\sqrt{\mu^{2}+\Delta^{2}}=$ $U / 2$ is independent of $T$. Therefore, both of these mean-field parameters not only are proportional to the binding energy $U$ of the two-body bound state in vacuum, but are also independent of $\alpha$ as the $\mathcal{T}$ symmetry ensures that the center of mass of the Cooper pairs is neutral against the flux. In other words, the only mechanism that allows a Cooper molecule to hop from one site to another is via the virtual breaking of its $\uparrow$ and $\downarrow$ constituents [22,23]. Since the cost for breaking the bound state is $U$, the molecule effectively hops from site $j$ to site $i$ with $t_{m i j}=2 t_{\uparrow i j} t_{\downarrow i j} / U$. Thus, for our nearest-neighbor lattice model, we identify $t_{m}=2 t^{2} / U$ as the hopping amplitude of the molecules and $\alpha_{m}=\alpha_{\uparrow}+\alpha_{\downarrow}=0$ as their flux.

In addition, this intuition further suggests that the SF density, and hence the SF phase stiffness, must be independent of $\alpha$ in the molecular limit. We prove this physical expectation by first approximating Eq. (8) as $D_{\mu \nu}=$ $\left[\Delta^{2} / \mathcal{A}\left(\mu^{2}+\Delta^{2}\right)^{3 / 2}\right] \sum_{n m \mathbf{k}} Q_{\mu \nu}^{n m \mathbf{k}}$ and then noting that the summation over $n$ and $m$ is equivalent to $\sum_{n m} Q_{\mu \nu}^{n m \mathbf{k}}=$ $\operatorname{Tr}\left[\left(\partial \mathbb{H}_{0 \mathbf{k}} / \partial k_{\mu}\right)\left(\partial \mathbb{H}_{0 \mathbf{k}} / \partial k_{\nu}\right)\right]$ for any given k. In particular to our Hamiltonian given in Eq. (2), we immediately get $4 t^{2} \sum_{\mathbf{k} \in \mathrm{MBZ}} \sum_{j=0}^{q-1} \sin ^{2}\left(k_{y} a+2 \pi \alpha j\right)$ for the $\mu \nu \equiv y y$ element and this summation is exactly equivalent to that of the flux-free system, i.e., $4 t^{2} \sum_{\mathbf{k} \in \mathrm{BZ}} \sin ^{2}\left(k_{y} a\right)=M / 2$, since the interval $-\pi \leqslant k_{y} a<\pi$ remains unchanged in both Brillouin zones. Thus, we conclude that $D_{0}=4 F(2-F) t^{2} / U$ is independent of both $\alpha$ and $T \leqslant T_{\mathrm{BKT}}$ in the molecular limit. It is worth 

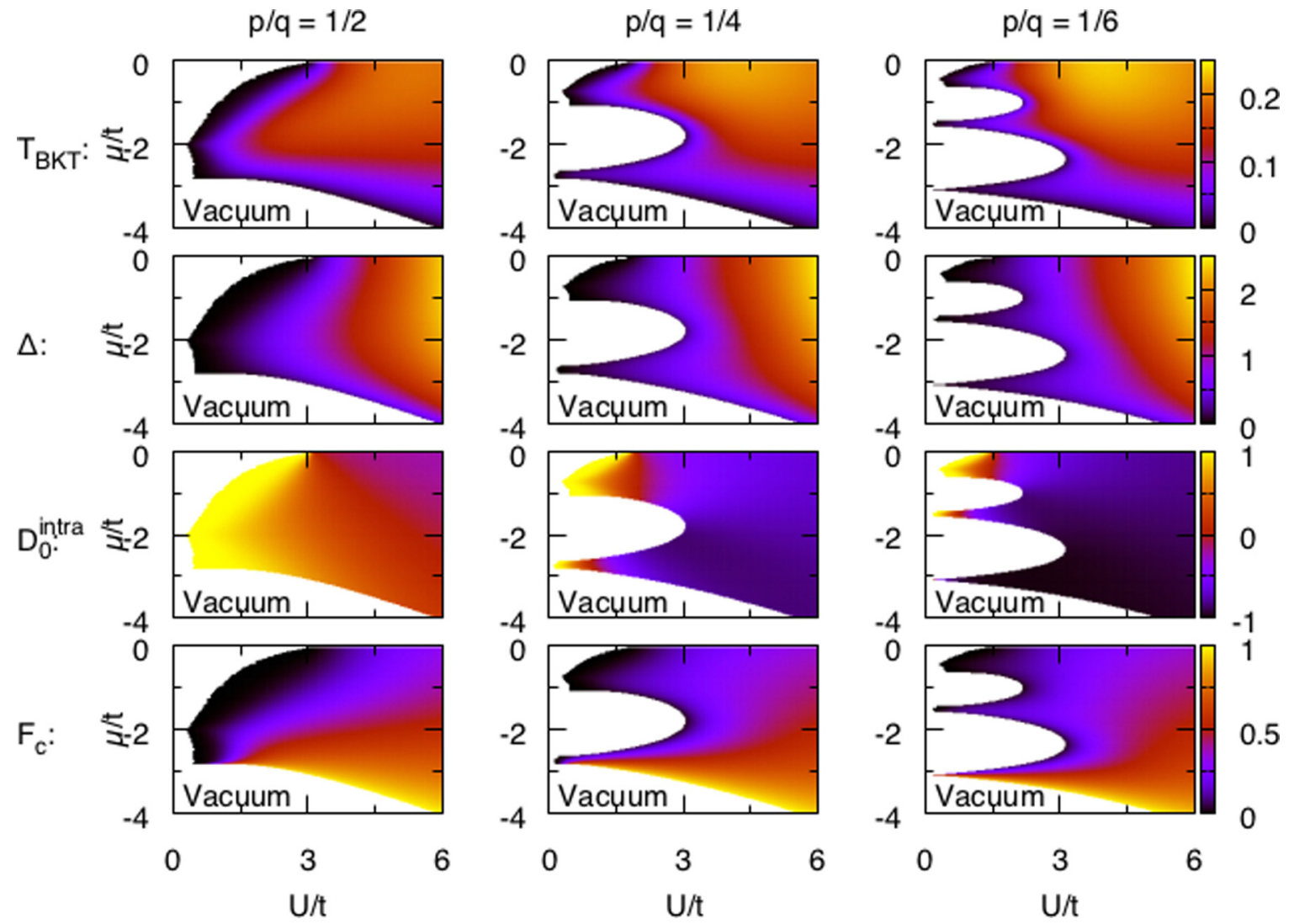

FIG. 1. The critical SF transition temperature $k_{B} T_{\mathrm{BKT}} / t$ is shown in the first row together with the corresponding SF order parameter $\Delta / t$ in the second row, the relative weight of the intraband and interband contributions to the phase stiffness $\left(D_{0}^{\text {intra }}-D_{0}^{\text {inter }}\right) / D_{0}$ in the third row, and the condensate fraction $F_{c} / F$ in the bottom row.

highlighting that, given the indiscriminate account of both the intraband and interband contributions in recovering the desired $D_{0}$ of the Cooper molecules, our proof offers indirect yet impartial support of the recent results. In fact, our numerical calculations presented in Sec. III reveal that $D_{0}^{\text {inter }}$ eventually dominates over $D_{0}^{\text {intra }}$ with increasing $U / t$ for any $q \geqslant 2$. While this domination is substantial even for the simplest two-band and three-band ( $q=2$ and 3 ) cases, it is already quite dramatic for $q>3$ as the butterfly bands get more flattened.

In addition, to make an analogy with the molecular limit of a continuum Fermi gas that is discussed at length in Sec. IIC, we first recall that the expression $D_{0}=\hbar^{2} \rho_{s} / m_{0}$ is derived for all $\Delta \neq 0$ including the molecular limit. Second, we rewrite $D_{0}$ in terms of the SF density of the continuum molecules $\rho_{\mathrm{sm}}=\rho_{s} / 2$ and their mass $m_{0 m}=2 m_{0}$ as $D_{0}=4 \hbar^{2} \rho_{\text {sm }} / m_{0 m}$. Then, by plugging the effective mass $m_{0 m}=\hbar^{2} / 2 t_{m} a^{2}$ of the lattice molecules into this continuum expression, we identify $F_{\text {sm }}=a^{2} \rho_{\text {sm }}=U D_{0} / 16 t^{2}=(F / 2)(1-F / 2)$ as the filling of SF molecules. We are able to confirm that $F_{\text {sm }}$ is independent of $\alpha$, which need not be the case for the filling of SF particles $F_{s}=a^{2} \rho_{s}=D_{0} / 2 t$ in the weak-coupling limit.

On the other hand, by adapting the definition of the number of condensed particles for our model [24],

$$
F_{c}=\frac{\Delta^{2}}{2 M} \sum_{n \mathbf{k}} \frac{\mathcal{X}_{n \mathbf{k}}^{2}}{E_{n \mathbf{k}}^{2}},
$$

and taking the molecular limit, we obtain $F_{\mathrm{cm}}=\Delta^{2} / 4\left(\mu^{2}+\right.$ $\left.\Delta^{2}\right)=(F / 2)(1-F / 2)$ as the filling of condensed molecules, which is also independent of $\alpha$. Thus, we conclude that all of the SF molecules are condensed with a fraction of $2 F_{\mathrm{sm}} / F=$ $2 F_{\mathrm{cm}} / F=1-F / 2$. In perfect agreement with the continuum model where we find that the entire Fermi gas is condensed and becomes a SF in the dilute $(F \rightarrow 0)$ limit, half of the Fermi gas is not condensed at half filling $(F \rightarrow 1)$. This difference between the dilute continuum and finite-filling lattice has to do with the fact that Cooper molecules are intrinsically hardcore by their composite nature, which is strictly dictated by the Pauli exclusion principle in the $U / t \rightarrow \infty$ limit. For this reason, whether a site is almost empty or singly occupied by one of the Cooper molecules gives rise to a notable outcome in lattice models.

\section{NUMERICAL RESULTS}

To illustrate the numerical accuracy of our analysis given in Sec. II, next we present the self-consistent solutions of Eqs. (4)-(6) for two sets of $\alpha=1 / q$ : The even $q \in\{2,4,6\}$ set is shown in Fig. 1 and the odd $q \in\{3,5,7\}$ is shown in Fig. 2. Here we primarily focus on the evolution of $T_{\mathrm{BKT}}$ together with the corresponding $\Delta, D_{0}^{\text {intra }}$, and $F_{c}$ in the $\mu$ versus $U$ plane. The trivial $q=1$ case is included in the Appendix for the sake of completeness. Due to the particle-hole symmetry of the model Hamiltonian, we restrict numerics to $\mu \leqslant 0$ or equivalently 


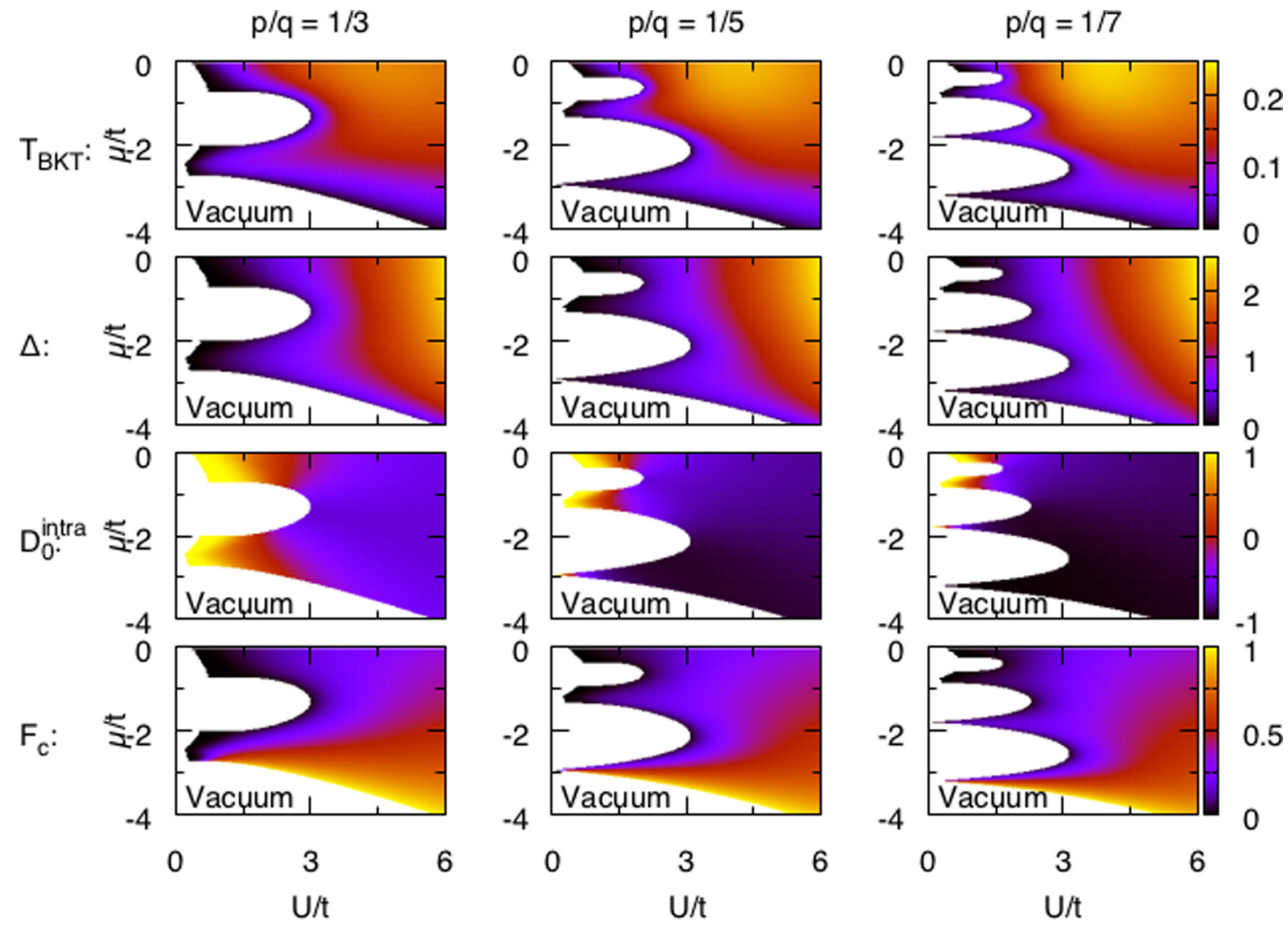

FIG. 2. The critical SF transition temperature $k_{B} T_{\mathrm{BKT}} / t$ is shown in the first row together with the corresponding SF order parameter $\Delta / t$ in the second row, the relative weight of the intraband and interband contributions to the phase stiffness $\left(D_{0}^{\text {intra }}-D_{0}^{\text {inter }}\right) / D_{0}$ in the third row, and the condensate fraction $F_{c} / F$ in the bottom row.

$F \leqslant 1$, as the solutions are mirror-symmetric around $\mu=0$ or the half filling $F=1$.

First of all, since $T_{\mathrm{BKT}} / t \rightarrow T_{c} / t \rightarrow 0$ as $\Delta / t \rightarrow 0$ or $U \rightarrow U_{c}$, where the value of the critical interaction threshold $U_{c}$ for SF pairing depends strongly on the energy density $\mathcal{D}(\varepsilon)$ of single-particle states, e.g., $U_{c} / t>0$ when $\mu$ lies within the butterfly gaps or at $\mu=0$ when $q$ is even, the top two rows in Figs. 1 and 2 recover the overall structure of the ground-state $(T \rightarrow 0)$ phase diagrams [18]. We recall that the multiband butterfly spectrum gives rise to a number of insulating lobes that are reminiscent of the well-known Mott-insulator transitions of the Bose-Hubbard model. This is because while $\Delta$, and therefore $T_{\mathrm{BKT}}$, grows exponentially $e^{-1 / U \mathcal{D}(\mu)}$ slow with $U \neq 0$ and $\mathcal{D}(\varepsilon)$ wherever $\mu$ lies within any of the butterfly bands, it grows linearly $U-U_{c}$ fast from the semimetal when $\mu=0$ and $q$ is even and with a square root $\sqrt{U-U_{c}}$ from the insulators in general [18,19].

Even though $\Delta$ and $T_{\mathrm{BKT}}$ must, in theory, vanish strictly at $U=0$ wherever $\mu$ lies within any of the butterfly bands, this appears not to be the case in Figs. 1 and 2, e.g., the $U \rightarrow 0$ regions appear white instead of black. This is due to a lack of our numerical resolution as the nonlinear solver fails to converge once the relative accuracy of two consecutive $\Delta$ iterations reduces below the order of $10^{-5}$. We checked that using a $10^{-6}$ resolution does not improve the phase diagrams, i.e., the minor corrections are indistinguishable to the eye. On the other hand, this shortage makes the general structure of $\mathcal{D}(\varepsilon)$ visible on the periphery of the white regions. In contrast, the insulating lobes are determined quite accurately, since $\Delta$ and $T_{\mathrm{BKT}}$ vanish very rapidly as $U \rightarrow U_{c} \neq 0$.

The top rows in Figs. 1 and 2 show that the maximum critical temperatures are always attained at $\mu=0$ for some intermediate $U \sim 3.75 t-5.5 t$ and are all of the order of $k_{B} T_{c}^{\max } \sim 0.19 t-0.25 t$ for any given $\alpha$. In particular, we approximately determine the following $\left(k_{B} T_{c}^{\max } / t, U / t\right)$ values in our numerics: $(0.1917,5.45)$ for $q=2,(0.2027,4.70)$ for $q=3,(0.2181,4.25)$ for $q=4,(0.2260,4.10)$ for $q=5$, $(0.2321,4.00)$ for $q=6$, and $(0.2363,3.95)$ for $q=7$. Thus, increasing $q$ from 2 not only enhances $\max k_{B} T_{c}^{\max } / t$ quite monotonically, but also occurs at a lower $U / t$. In comparison, we find $(0.2528,3.75)$ for $q=\infty$ or equivalently $q=1$ corresponding to the usual no-flux model presented in the Appendix. Assuming that the monotonic trend continues for larger $q$, we suspect that the result of the $q=\infty$ case is an ultimate upper bound for $T_{c}^{\max }$ in the entire parameter range of the model Hamiltonian given in Eq. (3). In the molecular limit when $U \gg t$, we verify that $k_{B} T_{\mathrm{BKT}} / t$ decreases as $\pi F(2-F) t / 2 U$ in all figures, which is in perfect agreement with the analysis given above in Sec. IID.

In addition, we present the relative $D_{0}^{\text {intra }}-D_{0}^{\text {inter }}$ weights of the intraband and interband contributions to $D_{0}$ in the third rows of Figs. 1 and 2. Together with the $T_{\mathrm{BKT}}$ figures 
shown in the top rows which are directly proportional to the sum $D_{0}^{\text {intra }}+D_{0}^{\text {inter }}$, these results reveal that $D_{0}^{\text {inter }}$ eventually dominates over $D_{0}^{\text {intra }}$ with increasing $U / t$ for any $q \geqslant 2$. While this domination is substantial even for the simplest two-band and three-band ( $q=2$ and 3 ) cases, it becomes sheer dramatic for $q>3$ once the butterfly bands get more flattened. Thus, our numerical results unveil and highlight the relative importance of $D_{0}^{\text {inter }}$ contribution without a doubt.

Finally, the condensate fractions $F_{c} / F$ are shown in the bottom rows of Figs. 1 and 2 . These results show that $F_{c} / F \rightarrow$ 0 is directly controlled by $\Delta$ in the weak-coupling limit when $\Delta / t \rightarrow 0$. On the other hand, $F_{c} / F$ saturates to $1-F / 2$ in the molecular limit, which is again in perfect agreement with the ground-state analysis given above in Sec. IID. This is because $k_{B} T_{\mathrm{BKT}} / t \rightarrow 0$ in both the $U \rightarrow U_{c}$ and $U \gg t$ limits. Having achieved the primary objectives of this paper, next we are ready to end it with a brief summary of our conclusions.

\section{CONCLUSION}

In summary, by studying the thermal SF properties along with the critical SF transition temperature in the HofstadterHubbard model with $\mathcal{T}$ symmetry, here we analyzed the competition between the intraband and interband contributions to the phase stiffness in the presence of a multiband butterfly spectrum. For instance, one of the highlights of this paper is that increasing the interaction strength always shifts the relative importance of the two in favor of the interband contribution. In marked contrast with the two-band and three-band cases for which the shift takes place gradually, our numerical results showed an extremely striking shift for the higher-band ones. We also showed analytically that the proper description of the Cooper molecules requires an indiscriminate account of both contributions in the strong-coupling limit.

Given our convincing evidence that the interband effects are absolutely non-negligible in a typical multiband butterfly spectrum, we hope to see further studies along this direction in other models and/or contexts as well. Presumably, similar to the resolution of the two-band superconductivity without supercurrent controversy near the Dirac points in graphene $[12,13]$, such effects may already be playing a part in the multiband family of high- $T_{c}$ superconductors that are waiting to be uncovered and characterized.

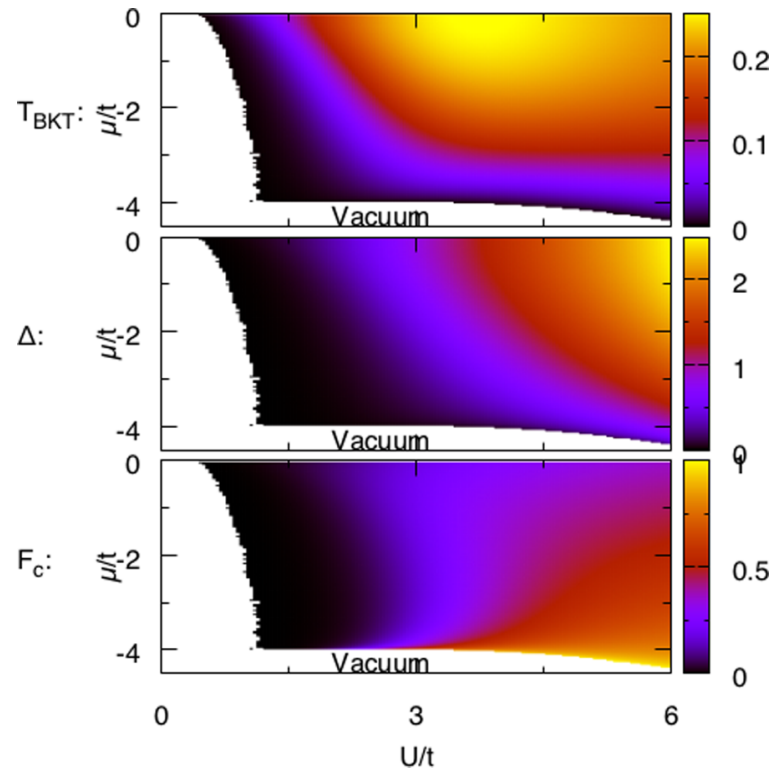

FIG. 3. The critical SF transition temperature $k_{B} T_{\mathrm{BKT}} / t$ is shown in the first row together with the corresponding SF order parameter $\Delta / t$ in the second row and the condensate fraction $F_{c} / F$ in the bottom row.

\section{ACKNOWLEDGMENTS}

The author acknowledges funding from TÜBITAK and the BAGEP award of the Turkish Science Academy.

\section{APPENDIX: USUAL HUBBARD MODEL}

For the sake of completeness, here we include the selfconsistent solutions of Eqs. (4)-(6) for $\alpha=0$ or equivalently $\alpha=1 / 1$, where $\varepsilon_{\mathbf{k}}=-2 t \cos \left(k_{x} a\right)-2 t \cos \left(k_{y} a\right)$. As we noted in Sec. III, even though $\Delta$ and $T_{\mathrm{BKT}}$ must, in theory, vanish strictly at $U=0$ wherever $\mu$ lies within the band, i.e., $-4 t<\mu<4 t$, this appears not to be the case in Fig. 3 as well, e.g., the $U \rightarrow 0$ regions appear white instead of black. This is again due to a lack of our numerical resolution as the nonlinear solver fails to converge once the relative accuracy of two consecutive $\Delta$ iterations reduces below the order of $10^{-5}$. Despite this shortage, we find $k_{B} T_{c}^{\max } \approx 0.2528 t$ at $\mu=0$ when $U \approx 3.75 t$, which is in very good agreement with an earlier estimate [4].
[1] M. E. Fisher, M. N. Barber, and D. Jasnow, Helicity modulus, superfluidity, and scaling in isotropic systems, Phys. Rev. A 8, 1111 (1973).

[2] D. J. Scalapino, S. R. White, and S. C. Zhang, Superfluid Density and the Drude Weight of the Hubbard Model, Phys. Rev. Lett. 68, 2830 (1992).

[3] D. J. Scalapino, S. R. White, and S. C. Zhang, Insulator, metal, or superconductor: The criteria, Phys. Rev. B 47, 7995 (1993).

[4] P. J. H. Denteneer, G. An, and J. M. J. van Leeuwen, Helicity modulus in the two-dimensional Hubbard model, Phys. Rev. B 47, 6256 (1993).
[5] V. L. Berezinskii, Destruction of long-range order in onedimensional and two-dimensional systems having a continuous symmetry group I. classical systems, Sov. Phys._JETP 32, 493 (1971).

[6] J. M. Kosterlitz and D. J. Thouless, Ordering, metastability and phase transitions in two-dimensional systems, J. Phys. C 6, 1181 (1973).

[7] D. R. Nelson and J. M. Kosterlitz, Universal Jump in the Superfluid Density of Two-Dimensional Superfluids, Phys. Rev. Lett. 39, 1201 (1977).

[8] S. Peotta and P. Törmä, Superfluidity in topologically nontrivial flat bands, Nat. Commun. 6, 8944 (2015). 
[9] A. Julku, S. Peotta, T. I. Vanhala, D.-H. Kim, and P. Törmä, Geometric Origin of Superfluidity in the Lieb-Lattice Flat Band, Phys. Rev. Lett. 117, 045303 (2016).

[10] L. Liang, T. I. Vanhala, S. Peotta, T. Siro, A. Harju, and P. Törmä, Band geometry, Berry curvature, and superfluid weight, Phys. Rev. B 95, 024515 (2017).

[11] L. Liang, S. Peotta, A. Harju, and P. Törmä, Wave-packet dynamics of Bogoliubov quasiparticles: Quantum metric effects, Phys. Rev. B 96, 064511 (2017).

[12] N. B. Kopnin and E. B. Sonin, BCS Superconductivity of Dirac Electrons in Graphene Layers, Phys. Rev. Lett. 100, 246808 (2008).

[13] N. B. Kopnin and E. B. Sonin, Supercurrent in superconducting graphene, Phys. Rev. B 82, 014516 (2010).

[14] M. Aidelsburger, M. Atala, M. Lohse, J. T. Barreiro, B. Paredes, and I. Bloch, Realization of the Hofstadter Hamiltonian with Ultracold Atoms in Optical Lattices, Phys. Rev. Lett. 111, 185301 (2013).

[15] C. J. Kennedy, G. A. Siviloglou, H. Miyake, W. C. Burton, and W. Ketterle, Spin-Orbit Coupling and Spin Hall Effect for Neutral Atoms without Spin-Flips, Phys. Rev. Lett. 111, 225301 (2013).

[16] D. R. Hofstadter, Energy levels and wave functions of Bloch electrons in rational and irrational magnetic fields, Phys. Rev. B 14, 2239 (1976).
[17] L. Wang, H.-H. Hung, and M. Troyer, Topological phase transition in the Hofstadter-Hubbard model, Phys. Rev. B 90, 205111 (2014).

[18] R. O. Umucalılar and M. Iskin, BCS Theory of Time-ReversalSymmetric Hofstadter-Hubbard Model, Phys. Rev. Lett. 119, 085301 (2017).

[19] M. Iskin, Hofstadter-Hubbard model with opposite magnetic fields: Bardeen-Cooper-Schrieffer pairing and superfluidity in the nearly flat butterfly bands, Phys. Rev. A 96, 043628 (2017).

[20] H. Zhai, R. O. Umucalılar, and M. Ö. Oktel, Pairing and Vortex Lattices for Interacting Fermions in Optical Lattices with a Large Magnetic Field, Phys. Rev. Lett. 104, 145301 (2010).

[21] R. O. Umucalılar and M. Iskin, Superfluid transition in the attractive Hofstadter-Hubbard model, Phys. Rev. A 94, 023611 (2016).

[22] P. Noziéres and S. Schmitt-Rink, Bose condensation in an attractive fermion gas: From weak to strong coupling superconductivity, J. Low Temp. Phys. 59, 195 (1985).

[23] L. Belkhir and M. Randeria, Collective excitations and the crossover from Cooper pairs to composite bosons in the attractive Hubbard model, Phys. Rev. B 45, 5087(R) (1992).

[24] A. J. Leggett, Quantum Liquids: Bose Condensation and Cooper Pairing in Condensed-Matter Systems (Oxford University Press, New York, 2006), Chap. 5. 